\title{
Reduced slow-wave sleep and altered diurnal cortisol rhythms in patients with Addison's disease
}

\author{
Michelle Henry ${ }^{1,2}$, lan Louis Ross ${ }^{3}$ and Kevin Garth Flusk Thomas ${ }^{1}$ \\ ${ }^{1}$ Department of Psychology, ACSENT Laboratory, ${ }^{2}$ Centre for Higher Education Development, and ${ }^{3}$ Division of \\ Endocrinology, Department of Medicine, University of Cape Town, Cape Town, South Africa
}

Correspondence should be addressed to M Henry

Email

m.henry@uct.ac.za

\begin{abstract}
Objectives: Cortisol plays a key role in initiating and maintaining different sleep stages. Patients with Addison's disease $(A D)$ frequently report disrupted sleep, and their hydrocortisone medication regimes do not restore the natural diurnal rhythm of cortisol. However, few studies have investigated relations between sleep quality, especially as measured by polysomnographic equipment, and night-time cortisol concentrations in patients with AD.

Methods: We used sleep-adapted EEG to monitor a full night of sleep in seven patients with AD and seven healthy controls. We sampled salivary cortisol before bedtime, at midnight, upon awakening and at 30 min post waking. Results: Controls had lower cortisol concentrations than patients before bedtime and at midnight. During the second half of the night, patient cortisol concentrations declined steeply, while control concentrations increased steadily. Whereas most controls experienced a positive cortisol awakening response, all patients experienced a decrease in cortisol concentrations from waking to $30 \mathrm{~min}$ post waking $(P=0.003)$. Patients experienced significantly lower proportions of slow-wave sleep (SWS; $P=0.001$ ), which was associated with elevated night-time cortisol concentrations.

Conclusion: Overall, these results suggest that patients with AD demonstrate different patterns of night-time cortisol concentrations to healthy controls and that relatively elevated concentrations are associated with a reduction of SWS. These hormonal and sleep architectural aberrations may disrupt the routine sleep-dependent processes of memory consolidation, and hence, may explain, at least partially, the memory impairments often experienced by patients with AD.
\end{abstract}

\section{Introduction}

Patients with Addison's disease (AD) have low plasma cortisol and aldosterone levels alongside high adrenocorticotropic hormone (ACTH) levels and renin concentrations. A standard pharmacological intervention for these patients thus involves administration of a cortisol replacement (most often oral hydrocortisone or prednisone), along with an additional mineralocorticoid (e.g., fludrocortisone) to control sodium and potassium balance (1).

There are reciprocal relationships between hormone secretion and regulation of the sleep-wake cycle $(2,3)$.
Hormones of the hypothalamic-pituitary-adrenal (HPA) axis play a particularly important role in facilitating entry into, and in the timing and duration of, various sleep stages. Healthy individuals experiencing normal diurnal rhythmicity show relatively low cortisol, ACTH and corticotrophin-releasing hormone (CRH) concentrations during the early part of the night, along with high melatonin and growth hormone-releasing hormone (GHRH) concentrations. The high proportion of SWS during the early part of the night is stimulated by GHRH, via its effects on growth hormone $(\mathrm{GH})$, and optimal

Published by Bioscientifica Ltd. 
cortisol levels experienced during early sleep probably enhance SWS through feedback inhibition of CRH (4, $5,6,7)$. In contrast, during the second half of the night, $\mathrm{CRH}$ stimulates cortisol and inhibits $\mathrm{GH}$, thus creating a physiological environment that is more conducive for entry into, and longer duration of, rapid eye movement (REM) sleep $(4,8)$.

Standard GC replacement therapy does not restore the normal cortisol diurnal rhythm in patients with $\mathrm{AD}$, resulting in alternating periods of sub- and supra-physiological concentrations. Hydrocortisone administration in the late afternoon or early evening leads to relatively elevated cortisol concentrations during the early part of the night (9), but the medication's short half-life results in cortisol deficiencies during the latter part of the night and the early morning (10). Given the importance of the HPA axis in sleep regulation (4), high night-time cortisol during the early hours of sleep, accompanied by high night-time ACTH and CRH, may be one reason why patients with $\mathrm{AD}$ frequently report experiencing disrupted, unrefreshing sleep $(11,12,13)$.

However, only two published studies have examined the relationship between HPA axis activity and objectively measured sleep in patients with $\mathrm{AD}(14,15)$. Although findings from both studies support the notion that HPA axis hypo- and hyperactivity in patients with $\mathrm{AD}$ alters sleep architecture, some methodological considerations temper any firm inferences. For instance, García-Borreguero et al. (14) did not report on the sleep architecture of their patients under basal conditions (i.e., when treated with usual replacement medication regimen), and Gillin et al. (15) was a small study in which patients $(n=3)$ and controls $(n=5)$ were not matched demographically (e.g. whereas patients' age range was 27-58, controls were aged $<25$ years).

Nonetheless, and especially in light of sizable literature describing relationships between irregular HPA axis activity and altered sleep architecture in both psychiatric and healthy samples $(16,17,18)$, it is plausible to suggest that hypo- or hyperactivity of that endocrinological system might play a role in explaining why patients with $\mathrm{AD}$ self-report, and objectively experience, poorer sleep quality and more frequent sleep disturbances than healthy controls $(11,12,14,19)$.

\section{The current study}

Given the known associations between cortisol secretion and sleep architecture, and the limited research on sleep in patients with $\mathrm{AD}$, we aimed to measure, using polysomnographic techniques, the sleep quality and architecture of patients with AD who were on immediaterelease hydrocortisone replacement therapy. Because we sought to maintain high levels of ecological validity, our design did not feature any manipulation of the patients' medication regimen; we describe possible mechanistic relations between cortisol concentrations and sleep architecture as they exist naturalistically, without any laboratory-based alteration, in this population.

We hypothesized that, compared to matched healthy controls, patients with $\mathrm{AD}$, possibly due to their alternating periods of sub- and supra-physiological cortisol concentrations, will experience poorer sleep quality and disrupted sleep architecture. We also hypothesized that patients will, relative to controls, experience higher cortisol concentrations during the first half of the night, but lower concentrations during the second. Finally, we explored associations between cortisol concentrations and sleep patterns.

\section{Subjects and methods}

\section{Participants}

Our sample was seven adult patients with AD (recruited from the South African Addison's disease database (SAAD)) (20) and seven healthy community-dwelling adults (recruited from a university community and surrounding neighborhoods). All were part of a cohort participating in a research program investigating sleep, cognition and quality of life in $\operatorname{AD}(11,19,21)$. We matched the groups on age and sex distribution (each group included two men and five women), and on level of education (all had completed high school).

To avoid sleep-confounding effects of age, psychiatric status and hormonal variations $(22,23,24)$, we included only (a) individuals between the ages of 18 and 55 years, (b) individuals who did not evince severe depressive symptomatology, (c) women who were pre-menopausal, not pregnant and not taking oral contraceptives. Healthy controls were required to be free of any chronic medical or psychiatric illnesses.

\section{Measures}

\section{Screening instruments}

A sociodemographic questionnaire obtained information about biographic variables and general medical history. For patients with $\mathrm{AD}$, we extracted from the SAAD 
database information regarding type and dosage of current medication and duration since diagnosis. The Mini International Neuropsychiatric Interview (MINI; English version 5.0.0) assessed current psychiatric status (25). The Beck Depression Inventory-Second Edition (BDI-II) measured current (2-week) intensity, severity and depth of depression (26). Individuals with BDI-II scores greater than 29 (indicating severe depression) were excluded from participation.

\section{Subjective sleep assessment}

The Pittsburgh Sleep Quality Index (PSQI) (27) measured self-reported sleep quality and disturbances over the month prior to completion. It comprises 19 items, each related to at least one of seven components (sleep quality, latency, duration, disturbances and efficiency; use of sleeping medication and daytime dysfunction due to disrupted sleep). The score on each component ranges from 0 to 3; hence, the total PSQI score ranges from 0 to 21 , with higher scores representing poorer sleep quality marked by more disruptions. Individuals with a total score above 5 are considered to have poor sleep quality (28).

\section{Objective sleep assessment}

We used a Nihon Kohden sleep-adapted electroencephalograph (EEG). PSGs are a reliable means of measuring sleep efficiency and total sleep time and of classifying sleep stages (29). In the current study, the PSG measured brain activity, eye movements and muscle activity as the participant entered and passed through different sleep stages. We recorded data following the International 10-20 system (30).

\section{Cortisol}

We took salivary cortisol measurements as surrogate indicators of plasma cortisol concentration, collecting samples using Sarstedt salivettes (Sarstedt, Nümbrecht, Germany). The samples were analyzed using a competitive electrochemiluminescent immunoassay on the Roche Cobas 6000 (Roche Diagnostics $\mathrm{GmbH}$ ) with a coefficient of variation of $4 \%$.

\section{Ethical approval and procedure}

Research Ethics Committees from the University of Cape Town's Department of Psychology and Faculty of Health Sciences, both of which adhere to the Declaration of Helsinki (31), approved the study procedures. All participants gave informed consent for collection, use and reporting of their data after being explained the full purpose of the study. No participant reported experiencing adverse events during the course of the study procedures. All data are held confidential, and participants' rights to anonymity and privacy were guaranteed and have been sustained.

We instructed those in the $\mathrm{AD}$ group to continue taking their hydrocortisone medication at their usual time and obtained verbal confirmation that they did so. All testing took place at a dedicated sleep laboratory. Sleep architecture was recorded in a sound-attenuated, lightand temperature-controlled room for two consecutive nights. The first of these was an adaptation night, which allowed participants to acclimatize to a new sleeping environment (29). Following precedent $(32,33,34)$, data from this night were not analyzed.

Each participant arrived at the hospital at $20: 30 \mathrm{~h}$ on the adaptation night. After reading and signing informed consent documents, they completed the PSQI and ESS, and were then attached to the PSG. At 22:00 h, lights were turned off and they were instructed to lie down and fall asleep as soon as possible. Lights were turned on at 06:00 h. Before leaving the laboratory, participants were instructed not to consume any caffeinated drinks during the day and to have their last meal $2-3 \mathrm{~h}$ before bedtime.

Participants returned to the sleep laboratory later that day, at around 20:00 h. The subsequent protocol was identical to that of the adaptation night, except that we sampled salivary cortisol four times (Fig. 1). Upon completion of these procedures, participants were debriefed and compensated.

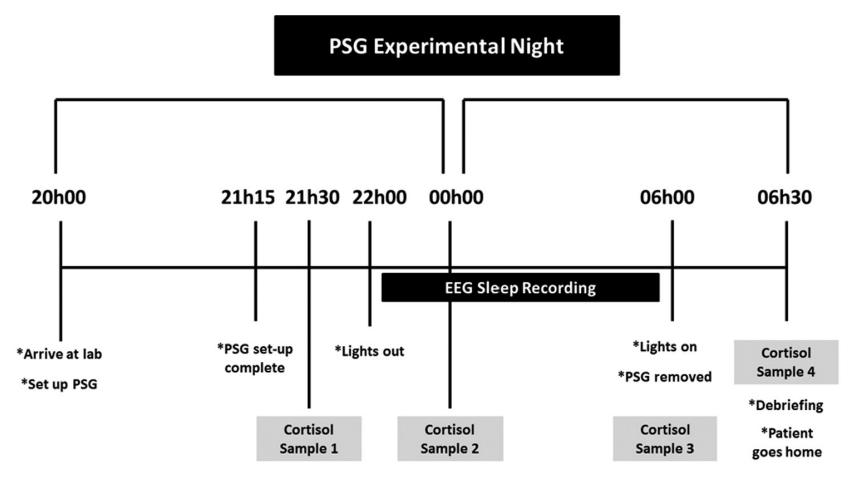

Figure 1

Study procedure during the experimental night. 


\section{Data management and statistical analyses}

\section{Subjective sleep}

We used PSQI total score to represent overall self-reported sleep quality and calculated the proportion of participants within each group who could be classified as poor sleepers (i.e., with total score $>5$ ). We also used individual PSQI items to estimate four other subjective sleep outcome variables: total sleep time (in minutes), number of minutes spent awake after sleep onset, sleep efficiency (proportion of time in bed spent asleep) and sleep latency (length of time, in minutes, between going to bed and falling asleep).

\section{Objective sleep}

On the experimental night, each participant was evaluated continuously for approximately $8 \mathrm{~h}$. We examined, for the full night and by half segments of the night, these 13 sleep-related outcome variables: sleep efficiency (proportion of time in bed spent asleep); sleep latency (length of time, in minutes, between lights out and falling asleep); REM latency (length of time, in minutes between lights out and onset of the first REM cycle); wake after sleep onset (WASO; the number of minutes spent awake in the period between sleep onset and final waking); time and proportion spent in each stage of sleep (N1, N2, SWS and REM) and number of awakenings (number of times the participant awoke for more than $1 \mathrm{~min}$ in the period between sleep onset and final waking).

We analyzed sleep data using Polysmith analysis software (Rosbach, Germany), and scored them according to standardized criteria outlined in the American Academy of Sleep Medicine Manual for the Scoring of Sleep and Associated Events (30). Sleep records were scored by M.H. and an independent qualified sleep technician, both of whom were blind to group assignment. For sleep-stage analysis, the two scorers had an epoch-by-epoch agreement of $95 \%$.

\section{Cortisol concentrations}

We used values from T1 (i.e., sample taken at 21:30 h), T2 (00:00 h), T3 (06:00 h), and T4 (06:30 h) to derive another three outcome variables: change in cortisol concentration during the first half of the night ( $\Delta$ CortFirst; T2-T1); change during the second half of the night ( $\Delta$ CortSecond; T3-T2) and cortisol awakening response (CAR; T4-T3).

\section{Power analysis and sample selection}

A priori power analyses suggested the sample size be set at $N=90$ (45 per group) to achieve statistical power of at least 0.95 using a cross-sectional matched-participant design investigating between-group differences (Cohen's $d=0.70 ; \alpha=0.05$ ) (35). We chose a medium-to-large effect size parameter because the only published investigation of baseline sleep architecture in $\mathrm{AD}$ patients reported an average Cohen's $d$ of 0.72 across all measured sleep variables (15). However, given the rarity of $\mathrm{AD}$, both globally and in South Africa (there are fewer than 200 patients in the SAAD database), along with our stringent eligibility criteria (e.g., fewer than 50 of the patients in the database met our age-related inclusion criterion), we could only enroll seven patients (and hence $N=14$ ). This sample size generated statistical power of 0.34 .

\section{Descriptive and inferential statistical analyses}

We completed all analyses using SPSS version 24 and $\mathrm{R}$ version 3.4.3, with the threshold for statistical significance $(\alpha)$ set at 0.05 unless noted otherwise. Given the small $N$ and non-normal distribution of outcome variables, we used a non-parametric statistical test (the Mann-Whitney $U$ test) to conduct between-group comparisons for (a) sociodemographic characteristics, (b) PSQI total score, (c) PSG-measured sleep quality and architecture for both the whole-night and split-night datasets and (d) cortisolrelated variables. We conducted a Fisher's exact test to determine the magnitude of association between group (AD vs Control) and proportion of individuals classified as poor sleepers (PSQI total score $>5$ ). A series of Wilcoxon signed-rank tests compared subjective and objective measures of total sleep time, WASO, sleep efficiency and sleep latency. Because of our small $N$, we used scatter plots to describe associations between (a) sleep variables and cortisol concentrations and (b) patient disease characteristics and sleep variables/cortisol concentrations.

\section{Results}

\section{Sample characteristics}

By design, there were no significant between-group differences in terms of age $(P=0.654)$ or education $(P=0.165$; Table 1). Analyses also detected no significant between-group BMI differences $(P=0.257)$.

\section{Patient clinical characteristics}

Two patients had hypothyroidism and one had diabetes. Structured psychiatric interviews suggested one could be 
Table 1 Overall sample characteristics $(n=14)$, and patient clinical characteristics $(n=7)$.

\begin{tabular}{l}
\hline Variable \\
\hline Age \\
Median (IQR) \\
Range \\
Education \\
Median (IQR) \\
Range \\
Body mass index ${ }^{\mathrm{a}}$ \\
Median (IQR) \\
Range \\
Age at diagnosis (years) \\
Mean (S.D.) \\
Range \\
Duration of AD (years) \\
Mean (S.D.) \\
Range \\
Total hydrocortisone dose (mg) \\
Mean (S.D.) \\
Range \\
Hydrocortisone (mg/kg) \\
Mean (S.D.) \\
Range \\
Number of doses per day \\
Mean (S.D.) \\
Range \\
Fludrocortisone dose (mg) ${ }^{\mathrm{d}}$ \\
Mean (S.D.) \\
Range \\
\hline
\end{tabular}

\begin{tabular}{c}
\hline Addison's disease $(n=7)$ \\
$40(23-44)$ \\
$20-55$ \\
$15(12-16)$ \\
$12-18$
\end{tabular}

$25.64(21.13-34.89)^{\mathrm{e}}$

20.05-37.55

$25.83(16.27)$

7-54

$10.00(4.82)$

1-14

$23.57(6.90)$

15-35

$0.30(0.10)$

$0.18-0.46$

$2.29(0.49)$

2-3

$0.14(0.38)$

$0.10-0.50$

Healthy controls $(n=7)$

$44(21-50)$

20-50

$12(12-15)$

12-15

23.24 (20.20-25.43)

19.26-26.37

ESE, effect size estimate (in this case, $r$ for Mann-Whitney $U$ tests).

${ }^{a}$ Calculated by dividing the participant's weight by height ${ }^{2}$ (information obtained from the sociodemographic questionnaire); ${ }^{b}$ Data based on 6 patients (1 patient did not provide the requisite information); ' $D$ ata based on 6 patients (1 patient did not provide weight-related information); ${ }^{\mathrm{d} D a t a}$ based on 5 patients ( 2 patients were not prescribed this medication); ${ }^{e}$ Data based on 4 participants ( 2 patients did not provide details of their height, and 1 did not provide details of her weight).

diagnosed with generalized anxiety disorder, agoraphobia and current manic episodes, and another two with generalized anxiety disorder. Tables 1 and 2 present further details regarding these characteristics, as well as individual regimens of immediate-release hydrocortisone.

\section{Between-group comparisons: subjective sleep data}

A larger proportion of patients than controls were classified as poor sleepers: four patients $(57.1 \%)$ and two controls (28.6\%) self-reported a PSQI total score $>5$, $P=0.296, V=0.29$. On average, patients reported poorer sleep quality (i.e., obtained higher PSQI total scores) than controls: AD: Median $(\mathrm{IQR})=6(3-8)$ vs Control: Median $(\mathrm{IQR})=3(2-6), U=15.5, P=0.242, r=0.31$.

\section{Between-group comparisons: objective sleep data}

Several extreme outliers ( $>3$ s.D.s from the mean) were present in the data (seven related to sleep outcome variables, two to cortisol outcome variables and one to patient timing of last daily dose; Supplementary Fig. 1, see section on supplementary data given at the end of this article). Results of analyses described below are reported with and without outliers.

Both patients and controls displayed the typical cyclical nature of sleep, with more SWS during the first half of the night and more REM sleep during the second half of the night. Of note is that the patient who took

Table 2 Patient medication regimen $(n=7)$.

\begin{tabular}{|c|c|c|c|c|}
\hline $\begin{array}{l}\text { Patient } \\
\text { number }\end{array}$ & $\begin{array}{l}\text { Total } \\
\text { dose } \\
(\mathrm{mg})\end{array}$ & $\begin{array}{c}\text { Dose } 1 \\
\text { (time, h) }\end{array}$ & $\begin{array}{c}\text { Dose } 2 \\
\text { (time, h) }\end{array}$ & $\begin{array}{l}\text { Dose } 3 \\
\text { (time, h) }\end{array}$ \\
\hline 1 & 15 & $10(08: 00)$ & $5(14: 00)$ & - \\
\hline 2 & 20 & $10(06: 30)$ & $5(11: 30)$ & $5(16: 00)$ \\
\hline 3 & 20 & $15(06: 00)$ & $5(17: 00)$ & - \\
\hline 4 & 20 & $10(07: 00)$ & $10(21: 00)$ & - \\
\hline 5 & 25 & $15(06: 00)$ & $5(12: 00)$ & $5(16: 00)$ \\
\hline 6 & 30 & $20(07: 00)$ & $10(16: 00)$ & - \\
\hline 7 & 35 & $25(07: 00)$ & $10(16: 00)$ & - \\
\hline
\end{tabular}


Table 3 Objective sleep quality and architecture in patients and controls $(n=14)$. Data are presented as median (IQR).

\begin{tabular}{l}
\hline Variable \\
\hline Whole night \\
Sleep efficiency \\
Sleep latency \\
REM latency \\
WASO \\
N1 time \\
N2 time \\
SWS time \\
REM time \\
N1\% \\
N2\% \\
N3\% \\
REM\% \\
Awakenings \\
First half of the night \\
Sleep efficiency \\
WASO \\
N1 time \\
N2 time \\
SWS time \\
REM time \\
N1\% \\
N2\% \\
N3\% \\
REM\% \\
Awakenings \\
Second half of the night \\
Sleep efficiency \\
WASO \\
N1 time \\
N2 time \\
SWS time \\
REM time \\
N1\% \\
N2\% \\
N3\% \\
REM\% \\
Awakenings \\
\hline
\end{tabular}

\begin{tabular}{c}
\hline Addison's disease $(n=7)$ \\
\hline $82.52(81.9-87.15)$ \\
$20.85(8.35-26.9)$ \\
$51.35(49.85-96.82)$ \\
$57.73(37.85-65.35)$ \\
$52.98(44.85-59.85)$ \\
$247.35(212.85-252.85)$ \\
$48.85(44.35-52.85)$ \\
$81.48(61.85-99.85)$ \\
$13.5(10.65-14.45)$ \\
$57.77(50.65-61.25)$ \\
$12.15(10.85-12.75)$ \\
$20.45(14.35-23.15)$
\end{tabular}

9.07 (5.85-10.85)

$79.5(77.5-83.9)$

$26.4(22.9-30.9)$

$22.9(19.4-32.4)$

122.9 (104.9-128.4)

33.4 (15.4-43.4)

$26.9(12.9-29.4)$

12 (9.4-16.6)

$61.4(52.9-62.9)$

$18.5(7.5-22.9)$

$12.8(9-13.5)$

$5.2(2.9-5.9)$

89.1 (81.2-94.8)

30.5 (11.9-45.4)

27.9 (21.9-31.4)

$120.9(109.9-121.9)$

$10.4(8.4-15.4)$

$58.1(32.4-75.4)$

$12.6(9.6-13.9)$

$55.3(51.6-61)$

$4.9(3.7-6.9)$

$26.7(16.3-32)$

$4.7(2.9-4.9)$

\begin{tabular}{c} 
Healthy controls $(n=7)$ \\
\hline \\
$83.47(79.37-87.4)$ \\
$15.85(10.35-32.35)$ \\
$142.82(82.35-206.35)$ \\
$65.85(51.35-72.35)$ \\
$55.98(45.85-66.85)$ \\
$185.32(163.35-206.85)$ \\
$75.82(56.35-93.35)$ \\
$92.35(55.35-108.35)$ \\
$14.85(11.25-18.15)$ \\
$47.35(40.15-49.15)$ \\
$19.55(13.75-20.45)$ \\
$22.42(14.95-23.85)$ \\
$8.85(6.85-10.07)$
\end{tabular}

$76.2(70.9-83.6)$

$29.9(23.9-43.9)$

$25.4(19.4-27.9)$

80.9 (73.4-94.9)

$55.7(52.9-57.4)$

$19.4(16.9-31.4)$

14.3 (13-15.9)

44.9 (39.7-45.7)

$28.6(27-33.1)$

$11.9(9.3-17)$

$4.9(3.9-5.7)$

90.6 (82.84-95.86)

$25.7(9.85-40.85)$

31.5 (18.85-42.35)

111.35 (87.85-115.35)

$21.1(0-35.85)$

$64.5(38.35-84.5)$

$15.3(7.9-20.4)$

$49(36.8-55.6)$

$9.8(0-14.9)$

$29.9(18.5-35.5)$

$2.85(1.85-4.85)$

2
2
2
1
1
2

.

$P$

$\begin{array}{lll}23 & 0.424 & 0.01\end{array}$

$\begin{array}{lll}22 & 0.375 & 0.09\end{array}$

110.042 * 0.46

$\begin{array}{lll}17 & 0.169 & 0.26\end{array}$

$\begin{array}{lll}17 & 0.283 & 0.15\end{array}$

$\begin{array}{lll}4 & 0.005^{*} & 0.70\end{array}$

$\begin{array}{lll}1.5 & 0.002 * * & 0.79\end{array}$

$\begin{array}{lll}20.5 & 0.305 & 0.14\end{array}$

$\begin{array}{lll}17 & 0.169 & 0.26\end{array}$

$\begin{array}{lll}3 & 0.004 * & 0.73\end{array}$

$0.5 \quad 0.001$ ** 0.82

$\begin{array}{lll}20 & 0.282 & 0.15\end{array}$

$\begin{array}{lll}21.5 & 0.350 & 0.10\end{array}$

$\begin{array}{lll}15.5 & 0.125 & 0.31\end{array}$

$\begin{array}{lll}17.5 & 0.185 & 0.24\end{array}$

$\begin{array}{lll}22 & 0.375 & 0.09\end{array}$

$\begin{array}{lll}6 & 0.009 * & 0.63\end{array}$

$<0.1 \quad 0.001 * * \quad 0.84$

$22 \quad 0.375 \quad 0.09$

$\begin{array}{lll}20 & 0.283 & 0.15\end{array}$

$4 \quad 0.005 * \quad 0.70$

$<0.1 \quad 0.001 * * \quad 0.89$

$\begin{array}{lll}24 & 0.475 & 0.02\end{array}$

$247 \quad 0.474 \quad 0.02$

${ }^{*} P<0.05 ;{ }^{*} P<0.004$ (statistically significant after the Bonferroni correction). ESE, effect size estimate (in this case, $r$ ); WASO, wake after sleep onset.

her last hydrocortisone dose latest in the day had the longest sleep latency (taking over an hour to fall asleep) and the shortest REM latency (less than $50 \mathrm{~min}$ ). For all participants, the brief awakenings at $\mathrm{T} 2$ had no detectable effect on sleep EEG. Results of between-group analyses are depicted in Table 3.

Across the whole night (Fig. 2) and during the first half of the night, patients with $\mathrm{AD}$ experienced, on average, significantly less SWS than controls. Analyses detected no significant between-group differences for measures pertaining to the second half of the night (all Ps $>0.055$ ). This pattern of results remained almost identical when data sets containing extreme outliers were removed from the analysis. The one exception here was that, in the outlier-free analysis, patients with AD had significantly more N2 sleep during the first half of the night than controls.

Within both groups, subjective sleep reports (for pastmonth sleep) were consistent with objective PSG-measured sleep on the experimental night (i.e., the analyses detected no significant between-measure differences; Table 4).

\section{Between-group comparisons: cortisol concentrations}

Analyses detected significant between-group differences at $\mathrm{T} 4$ (patients had significantly lower cortisol levels $30 \mathrm{~min}$ post awakening) and for CAR (Table 5). Of note regarding the latter is that five of the six control participants for 

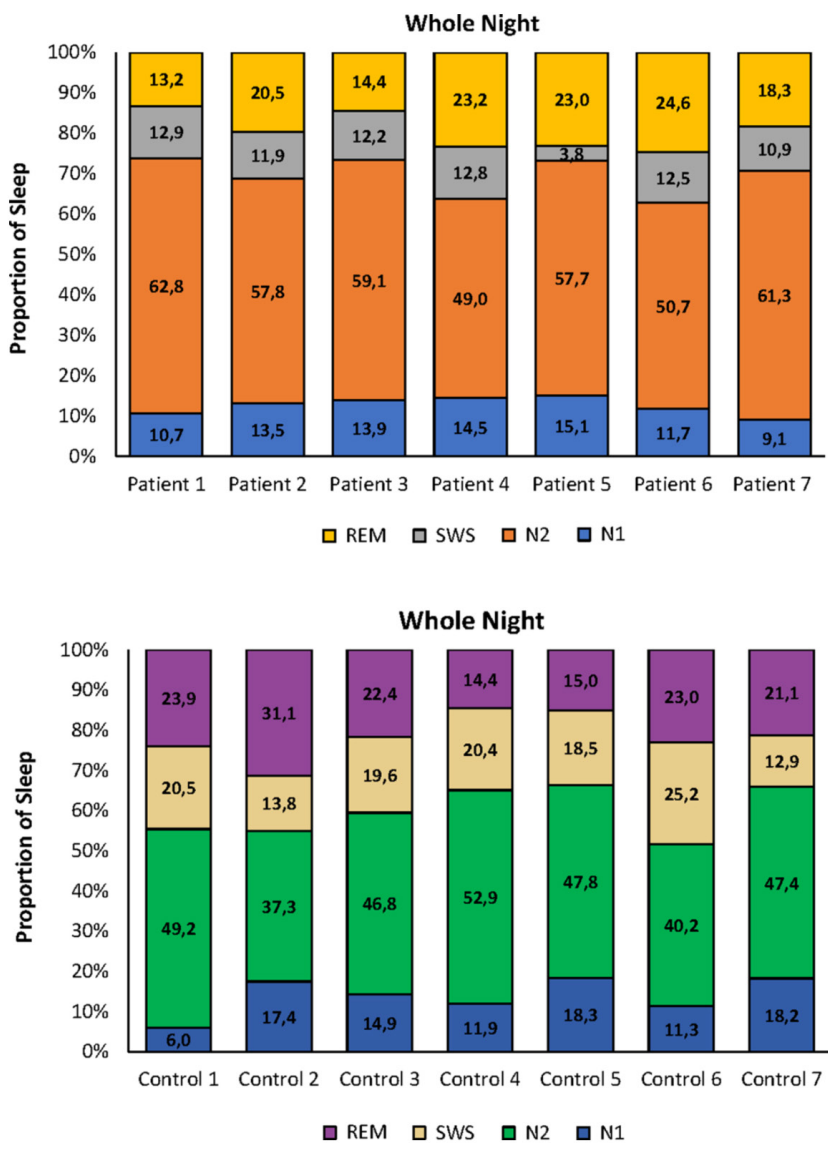

Figure 2

Distribution of sleep stages across the whole night, for each patient and control.

whom the variable could be calculated experienced a positive CAR, whereas none of the patients did. Furthermore, whereas controls experienced an increase in cortisol levels during the second half of the night, patients experienced a decrease. This pattern of results held when extreme outliers were removed from the data.

The pattern of cortisol concentrations was relatively homogenous for controls, but more variable for patients (Fig. 3). Of note is that, compared to all other patients, patient 4 (i.e., the individual who took her last hydrocortisone dose latest in the day) had a substantially higher cortisol concentration at bedtime and experienced the biggest decline in concentrations during the second half of the night.

\section{Correlations: sleep and cortisol}

We described associations, within each group separately, between sleep in each half of the night and specific cortisol values. Because three controls had missing cortisol values at midnight, we do not describe relationships of sleep outcomes with cortisol values at midnight, or with $\Delta$ CortFirst and $\Delta$ CortSecond, in this group.

\section{Sleep during the first half of the night and cortisol concentrations}

Here, we describe associations between PSG outcome variables, taken during the first half of the night, and three cortisol outcome variables: (a) the measure taken at bedtime, (b) the measure taken at midnight and (c) $\Delta$ CortFirst. Within the AD group, patients with higher cortisol concentrations at bedtime had fewer awakenings, and patients with higher cortisol levels at midnight had less SWS. When outliers were removed, only the association between cortisol levels at midnight and SWS remained.

Within the healthy control group, those with higher cortisol concentrations at bedtime had shorter REM latencies, better sleep efficiency and less SWS. Generally, this pattern of results held when outliers were removed; the only exception was that cortisol levels at bedtime and sleep efficiency were no longer associated.

\section{Sleep during the second half of the night and cortisol concentrations}

Here, we described associations between PSG outcome variables, taken during the second half the night, and three cortisol outcome variables: (a) the measure taken

Table 4 Comparison of subjective and objective sleep measures in patients and controls $(n=14)$. Data are presented as median (IQR).

\begin{tabular}{|c|c|c|c|c|c|}
\hline \multirow[b]{2}{*}{ Variable } & \multicolumn{5}{|c|}{ Addison's disease $(n=7)$} \\
\hline & PSQI & PSG & $z$ & $P$ & ESE \\
\hline TST (h) & $7(7-7.5)$ & $6.93(6.71-7.12$ & -0.34 & .735 & 0.09 \\
\hline WASO (min) & $42(30-90)$ & $57.73(37.85-65.35)$ & -0.85 & .398 & 0.23 \\
\hline Latency (min) & $10(5-30)$ & $20.85(8.35-26.90)$ & -0.68 & .499 & 0.18 \\
\hline Efficiency (\%) & $90.32(70-96.77)$ & $82.52(81.90-87.15)$ & -0.68 & .499 & 0.18 \\
\hline
\end{tabular}

\begin{tabular}{|c|c|c|c|c|}
\hline \multicolumn{5}{|c|}{ Control $(n=7)$} \\
\hline PSQI & PSG & $z$ & $P$ & ESE \\
\hline $7(6.5-8)$ & $6.59(6.37-7.26)$ & -0.34 & .735 & 0.09 \\
\hline $35(0-50)$ & $65.85(51.35-72.35)$ & -1.86 & .063 & 0.50 \\
\hline $10(4-15)$ & $15.85(10.35-32.35)$ & -1.86 & .063 & 0.50 \\
\hline $86.67(80-89)$ & $83.47(79.37-87.40)$ & -0.68 & .499 & 0.18 \\
\hline
\end{tabular}

ESE, effect size estimate (in this case, $r$ for Wilcoxon signed-rank tests); TST, total sleep time; WASO, wake after sleep onset. 
Table 5 Cortisol concentrations in patients and controls $(n=14)$. Data are presented as median (IQR).

\begin{tabular}{l} 
Variable \\
\hline T1 $(21: 30 \mathrm{~h})$ \\
T2 $(00: 00 \mathrm{~h})$ \\
T3 $(06: 00 \mathrm{~h})$ \\
T4 $(06: 30 \mathrm{~h})$ \\
$\Delta$ CortFirst \\
$\Delta$ CortSecond \\
CAR
\end{tabular}

\begin{tabular}{c}
\hline Addison's disease $(n=7)$ \\
\hline $8.02(5.91-10.29)$ \\
$13.98(11.01-20.29)$ \\
$12.62(7.22-16.14)^{\mathrm{a}}$ \\
$6.70(4.32-10.41)$ \\
$5.49(1.37-11.92)$ \\
$-1.45(-6.27 \text { to } 2.90)^{\mathrm{a}}$ \\
$-5.00(-8.75$ to -2.50$)$
\end{tabular}

\begin{tabular}{c}
\hline Healthy controls $(n=7)$ \\
\hline $5.28(4.58-6.26)^{\mathrm{b}}$ \\
$7.30(6.67-8.73)^{\mathrm{c}}$ \\
$12.32(10.99-20.24)^{\mathrm{d}}$ \\
$21.67(14.37-25.58)$ \\
$2.41(0.74-4.15)^{\mathrm{c}}$ \\
$5.50(3.71-7.53)^{\mathrm{c}}$ \\
$3.50(2.00-8.25)^{\mathrm{d}}$
\end{tabular}

$\begin{array}{r}\hline \boldsymbol{U} \\ \hline 5 \\ 1 \\ 12 \\ 4 \\ 7 \\ 1 \\ 0.5 \\ \hline\end{array}$

\begin{tabular}{l}
\hline \multicolumn{1}{c}{} \\
\hline $0.011 *$ \\
$0.007 *$ \\
0.169 \\
$0.002 * *$ \\
0.093 \\
$0.009 *$ \\
$0.003 * *$ \\
\hline
\end{tabular}

\begin{tabular}{l} 
ESE \\
\hline 0.64 \\
0.74 \\
0.28 \\
0.77 \\
0.40 \\
0.74 \\
0.78
\end{tabular}

${ }^{a}$ Data based on six participants (1 sample returned as insufficient for analysis); ${ }^{b}$ data based on six participants ( 1 sample returned as insufficient for analysis); ' data based on four participants ( 2 samples returned as insufficient for analysis; one sample was not obtained due to researcher error); ${ }^{d}$ data based on six participants ( 1 sample returned as insufficient for analysis); ${ }^{2}<0.05 ; * * P<0.007$ (statistically significant after the Bonferroni correction). CAR, cortisol awakening response; ESE, effect size estimate (in this case, $r$ for Mann-Whitney $U$ tests).

at midnight, (b) the measure taken upon awakening and (c) $\Delta$ CortSecond. Within the $\mathrm{AD}$ group, patients with higher cortisol concentrations at waking had better sleep efficiency and those who had larger decreases during the second half of the night experienced more SWS. When outliers were removed, only the association between cortisol levels upon awakening and sleep efficiency remained.

Within the healthy control group, those with higher cortisol concentrations at waking had more REM sleep. This association remained after outliers were removed.

\section{Correlations: patient disease characteristics, whole- night sleep and cortisol}

Because nearly all patients took two doses of hydrocortisone medication per day (bar two patients who took three doses/day), we did not examine the relationships between number of doses and sleep/cortisol variables. Patients with a higher total hydrocortisone dose had poorer sleep efficiency and those with a higher dose $/ \mathrm{kg}$ had longer sleep latencies and less N2\%. Patients who took their last dose of medication later in the day had less N3\%, higher cortisol levels at bedtime and a larger decrease in cortisol during the second half of the night. When outliers were removed, only the association between dose $/ \mathrm{kg}$ and $\mathrm{N} 2 \%$ remained, however.

Duration of AD appeared to bear no substantial association to any sleep or cortisol outcome variable.

\section{Discussion}

Several studies document self-reported disturbances of sleep quality in patients with $\mathrm{AD}$, but few have used polysomnographic measures to confirm these disruptions. The current study aimed to quantify sleep quality and architecture of patients with $\mathrm{AD}$ and to explore the role of cortisol concentrations in altered sleep patterns. Our patient group exhibited altered sleep architecture (specifically, reduced SWS), which was associated with elevated night-time cortisol concentrations.

Our results are consistent with the only other study in the AD literature that reports on PSG-measured sleep when replacement medication is administered as usual (15). Across the two studies, patients spent similar proportions of time in Stage 2 sleep (63\% in the current study vs $57 \%$ in Gillin et al. (15)), in SWS (14\% vs 11\%) and in REM sleep (19\% vs $20 \%)$. Moreover, in that study, as in ours, patients exhibited similar sleep quality and architecture to healthy controls, aside from SWS disruptions. As noted earlier, however, Gillin et al. (15) did not include matched samples of patients and controls.

SWS plays a critical role in the general physiological restorative function of sleep and in specific cognitive processes such as memory consolidation $(36,37)$. Hence, disrupted sleep and altered circadian rhythms lead to energy imbalances, fatigue, memory deficits and poorer quality of life $(38,39)$. Therefore, it is likely that when patients with AD experience decreased SWS they will also experience these negative health outcomes. Indeed, fatigue and memory deficits are features of adrenal failure that persist despite replacement therapy and are major contributors to self-reported impaired health in $\mathrm{AD}(19,40)$.

Regarding between-group differences in night-time cortisol concentrations, patients had higher cortisol levels prior to sleep onset and during the first half of the night, but cortisol deficiencies during the second half of the night. The most marked between-group difference occurred after waking: Whereas most controls experienced a positive CAR, no patient did. These data are consistent with previous reports suggesting that, despite GC replacement therapy, patients with $\mathrm{AD}$ experience 

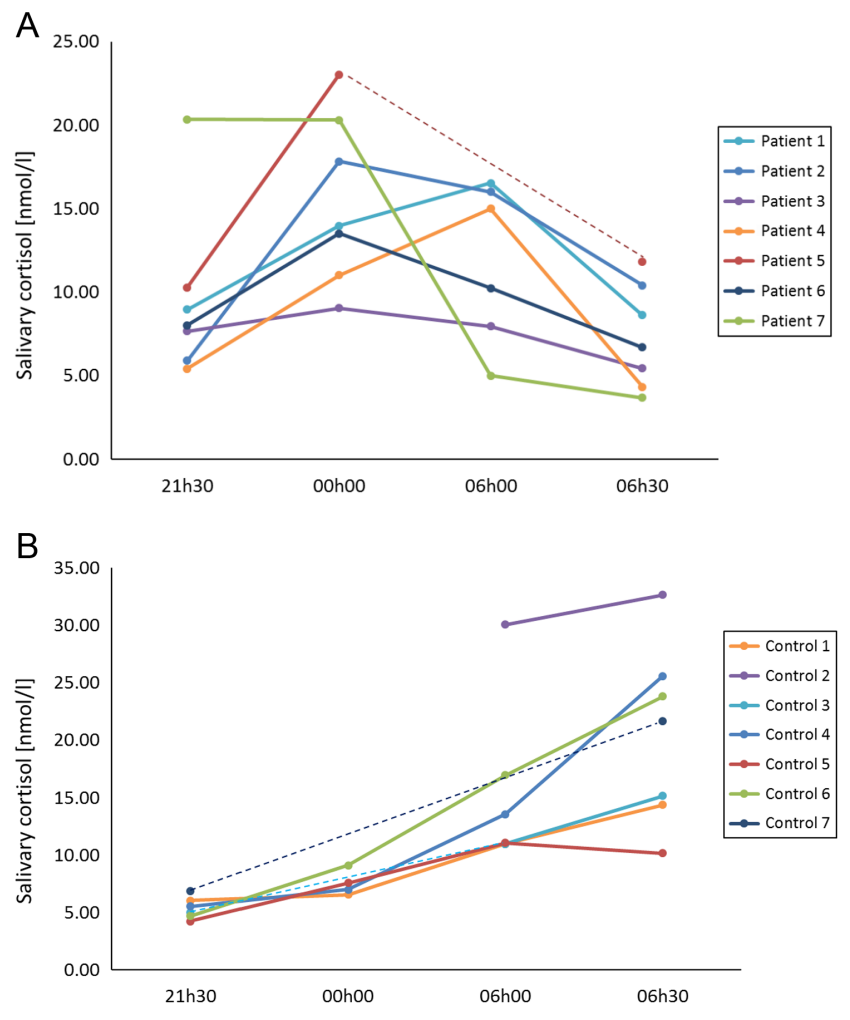

Figure 3

Changes in cortisol levels across the night and morning for each patient (A) and control (B). Data for one patient and three controls were not available at various time points - their graphs have dashed lines representing the potential trend in cortisol level changes (except for Control 2 who is missing the first two cortisol measurements and therefore a trend estimation cannot be made).

alterations of normal cortisol diurnal rhythms (41). Moreover, the observed trends during the latter part of the night and early morning are consistent with previous literature reporting on cortisol secretory action in patients with AD (10). Because cortisol plays a vital role in ensuring smooth transitions between sleep stages (42), irregular patterns of secretion in patients with AD might explain their altered sleep architecture.

Consistent with this conjecture, between-group differences in sleep architecture and in cortisol concentrations both occurred during the first half of the night, and our scatter plot analyses confirmed associations between levels of the hormone and discrete sleep parameters. For instance, within the $\mathrm{AD}$ group, higher cortisol concentrations at midnight were associated with less SWS, whereas lower cortisol concentrations during the second half of the night were associated with more SWS. These results are consistent with a substantial body of research indicating that high cortisol concentrations during the first half of the night (as exhibited by our patient group) are associated with less SWS (also exhibited by our patient group; i.e., lower cortisol levels facilitate the initiation and maintenance of SWS $)(2,15)$. Similarly, controls who had higher cortisol concentrations at bedtime had less SWS. Within the control group, higher cortisol concentrations at bedtime were associated with shorter REM latency, a finding consistent with research indicating that higher cortisol levels facilitate entry into REM sleep $(8,14,43)$.

In the current sample, some disease and medication characteristics of patients with $\mathrm{AD}$ were associated with sleep parameters and night-time cortisol secretion. Patients who took larger doses of hydrocortisone had poorer sleep efficiency, shorter sleep latency and less Stage 2 sleep. This result is understandable given that larger doses should translate into higher cortisol concentrations, and high cortisol concentrations increase both sleep onset latency and number of awakenings after sleep onset (2). Patients who took their last dose of hydrocortisone later in the day had higher cortisol concentrations at bedtime and less SWS, further supporting the notion that low cortisol levels are needed to facilitate the initiation and maintenance of SWS.

\section{Limitations and future directions}

Measurement and sample size issues limit the strength of the conclusions we can draw from the current data. The fact that we took only four saliva samples from bedtime through waking means we are limited in making inferences about how subtle changes in nocturnal cortisol secretory patterns, particularly between midnight and early morning when REM sleep predominates, may influence sleep parameters. Future studies might test the proposition that SWS is more sensitive to cortisol alterations than REM.

Furthermore, although cortisol measurement by saliva has numerous advantages over that by plasma (e.g., stress-free sampling, lower costs, and non-invasive collection methods) (44), salivary cortisol measured by radioimmunoassay has intrinsic problems of variability. In the current sample, for instance, concentrations may have been influenced by contamination of saliva with hydrocortisone tablets (and may explain, for instance, why patients experienced increasing cortisol concentrations between $21: 30 \mathrm{~h}$ and midnight, despite taking their final medication dose several hours prior to bedtime and the relatively short half-life of hydrocortisone). Given this 
uncertainty surrounding radioimmunoassay evaluation of salivary cortisol, future analyses should use liquid chromatography tandem mass spectrometry. Given the unusual finding that cortisol concentrations increased from bedtime to midnight in both patients and controls, further studies are needed to confirm the associations we found between sleep architecture and cortisol levels.

Furthermore, the fact that we gathered sleep data in a laboratory setting, over one night, means the sleep quality/architecture we analyzed may not accurately reflect participants' normal home-based sleep patterns (45). Although our analyses comparing subjective to objective sleep measures suggested that the patterns we captured in the laboratory were similar to those participants reported experiencing at home, future research in the field might take polysomnographic measures of sleep in a more naturalistic environment and over more nights.

Regarding sample size issues, our small $N$ meant that, for instance, we could not investigate the influence of biological sex on sleep quality/architecture in patients with $\mathrm{AD}$ or the influence of certain disease characteristics on sleep and cortisol measures. It should be noted that time of last dose was relatively homogenous (6 of the seven patients took their last dose between 14:00 and 17:00 h), and only two patients had any medical and psychiatric comorbidities. Therefore, any potential investigation of these variables was limited by the characteristics of the current sample.

A fruitful direction for future research might be to experimentally manipulate medication dosage and/or timing in patients with $\mathrm{AD}$ and to take repeated measures with each manipulation, thus allowing each patient to act as his/her own control. This design would allow for a true experimental test of the hypothesis that altered cortisol levels impact sleep architecture and would help control for duration of AD diagnosis in individual patients. Similarly, future research might investigate whether modifiedrelease hydrocortisone (which results in cortisol circadian rhythms that are more similar to those presents in healthy adults) improves sleep in patients with AD.

\section{Summary and conclusion}

Relative to healthy controls, patients with $\mathrm{AD}$ who were on standard hydrocortisone replacement therapy exhibited altered cortisol diurnal rhythms, accompanied by decreases in SWS. Because SWS plays a critical role in the general physiological restorative function of sleep and in memory consolidation, disrupted sleep in patients with AD may help explain why patients often report and experience fatigue, reduced vitality, poorer health and memory impairments. Hence, interventions focused on treating sleep disruptions in patients with AD might be especially helpful in improving specific aspects of their cognitive functioning and their overall quality of life.

\section{Supplementary data}

This is linked to the online version of the paper at https://doi.org/10.1530/ EJE-18-0439.

\section{Declaration of interest}

The authors declare that there is no conflict of interest that could be perceived as prejudicing the impartiality of this study.

\section{Funding}

This research did not receive any specific grant from any funding agency in the public, commercial or not-for-profit sector.

Author contribution statement

M H and K G F T designed the study; $\mathrm{M} \mathrm{H}$ collected the data; $\mathrm{M} \mathrm{H}$ and $\mathrm{K}$ G F T analyzed the data and interpreted the results; all authors prepared the manuscript.

Acknowledgments

The authors wish to thank all participants for their time and commitment to this research.

\section{References}

1 Ten S, New M \& Maclaren N. Addison's disease 2001. Journal of Clinical Endocrinology and Metabolism 200186 2909-2922. (https:// doi.org/10.1210/jcem.86.7.7636)

2 Steiger A. Sleep and endocrine regulation. Frontiers in Bioscience 2003 8 s358-s376. (https://doi.org/10.2741/1055)

3 McEwen BS \& Karatsoreos IN. Sleep deprivation and circadian disruption: stress, allostasis, and allostatic load. Sleep Medicine Clinics 201510 1-10. (https://doi.org/10.1016/j.jsmc.2014.11.007)

4 Buckley TM \& Schatzberg AF. On the interactions of the hypothalamic-pituitary-adrenal (HPA) axis and sleep: normal HPA axis activity and circadian rhythm, exemplary sleep disorders. Journal of Clinical Endocrinology and Metabolism 200590 3106-3114. (https:// doi.org/10.1210/jc.2004-1056)

5 Perras B, Marshall L, Kohler G, Born J \& Fehm HL. Sleep and endocrine changes after intranasal administration of Growth Hormone-releasing hormone in young and aged humans. Psychoneuroendocrinology 199924 743-757. (https://doi.org/10.1016/ S0306-4530(99)00027-X)

6 Steiger A, Guldner J, Hemmeter U, Rothe B, Wiedemann K \& Holsboer F. Effects of growth hormone-releasing hormone and somatostatin on sleep EEG and nocturnal hormone secretion in male controls. Neuroendocrinology 199256 566-573. (https://doi. org/10.1159/000126275)

7 Schier T, Guldner J, Colla M, Holsboer F \& Steiger A. Changes in sleep endocrine activity after growth hormone-releasing hormone depend on time of administration. Journal of Neuroendocrinology 1997 201-205. 
8 Vgontzas AN, Bixler EO, Papanicolaou DA, Kales A, Stratakis CA, Vela-Bueno A, Gold PW \& Chrousos GP. Rapid eye movement sleep correlates with the overall activities of the hypothalamic-pituitaryadrenal axis and sympathetic system in healthy humans. Journal of Clinical Endocrinology and Metabolism 199782 3278-3280. (https:// doi.org/10.1210/jcem.82.10.4307)

9 Ross IL, Levitt NS, Van der Walt JS, Schatz DA, Johannsson G, Haarburger DH \& Pillay TS. Salivary cortisol day curves in Addison's disease in patients on hydrocortisone replacement. Hormone and Metabolic Research 201345 62-68. (https://doi. org/10.1055/s-0032-1321855)

10 Harbeck B, Kropp P \& Mönig H. Effects of short-term nocturnal cortisol replacement on cognitive function and quality of life in patients with primary or secondary adrenal insufficiency: a pilot study. Applied Psychophysiology and Biofeedback 200934 113-119. (https://doi.org/10.1007/s10484-009-9082-5)

11 Henry M, Wolf PS, Ross IL \& Thomas KG. Poor quality of life, depressed mood, and memory impairment may be mediated by sleep disruption in patients with Addison's disease. Physiology and Behavior 2015151 379-385. (https://doi.org/10.1016/j. physbeh.2015.08.011)

12 Lovas K, Husebye ES, Holsten F \& Bjorvatn B. Sleep disturbances in patients with Addison's disease. European Journal of Endocrinology 2003148 449-456. (https://doi.org/10.1530/eje.0.1480449)

13 Øksnes M, Björnsdottir S, Isaksson M, Methlie P, Carlsen S, Nilsen RM, Broman J-E, Triebner K, Kämpe O, Hulting A-L et al. Continuous subcutaneous hydrocortisone infusion versus oral hydrocortisone replacement for treatment of Addison's disease: a randomized clinical trial. Journal of Clinical Endocrinology and Metabolism 201499 1665-1674. (https://doi.org/10.1210/jc.20134253)

14 Garcĺa-Borreguero D, Wehr TA, Larrosa O, Granizo JJ, Hardwick D, Chrousos GP \& Friedman TC. Glucocorticoid replacement is permissive for rapid eye movement sleep and sleep consolidation in patients with adrenal insufficiency 1. Journal of Clinical Endocrinology and Metabolism 200085 4201-4206. (https://doi.org/10.1210/ jcem.85.11.6965)

15 Gillin JC, Jacobs LS, Snyder F \& Henkin RI. Effects of decreased adrenal corticosteroids: changes in sleep in normal subjects and patients with adrenal cortical insufficiency. Electroencephalography and Clinical Neurophysiology 197436 283-289. (https://doi. org/10.1016/0013-4694(74)90170-9)

16 Backhaus J, Junghanns K, Born J, Hohaus K, Faasch F \& Hohagen F. Impaired declarative memory consolidation during sleep in patients with primary insomnia: influence of sleep architecture and nocturnal cortisol release. Biological Psychiatry 200660 1324-1330. (https://doi. org/10.1016/j.biopsych.2006.03.051)

17 Dijk D-J \& Lockley SW. Invited review: integration of human sleep-wake regulation and circadian rhythmicity. Journal of Applied Physiology 200292 852-862. (https://doi.org/10.1152/ japplphysiol.00924.2001)

18 Wulff K, Gatti S, Wettstein JG \& Foster RG. Sleep and circadian rhythm disruption in psychiatric and neurodegenerative disease. Nature Reviews: Neuroscience 201011 589-599. (https://doi. org/10.1038/nrn2868)

19 Henry M, Ross IL, Wolf PSA \& Thomas KGF. Impaired quality and efficiency of sleep impairs cognitive functioning in Addison's disease. Psychoneuroendocrinology 201778 237-245. (https://doi. org/10.1016/j.psyneuen.2017.02.004)

20 Ross I, Boulle A, Soule S, Levitt N, Pirie F, Karlsson A, Mienie J, Yang P, Wang H \& She JX. Autoimmunity predominates in a large South African cohort with Addison's disease of mainly European descent despite long-standing disease and is associated with HLA DQB*. Clinical Endocrinology 201073 291-298.

21 Henry M, Thomas KGF \& Ross IL. Episodic memory impairment in Addison's disease: results from a telephonic cognitive assessment.
Metabolic Brain Disease 201429 421-430. (https://doi.org/10.1007/ s11011-014-9511-x)

22 Kirschbaum C, Kudielka BM, Gaab J, Schommer NC \& Hellhammer DH. Impact of gender, menstrual cycle phase, and oral contraceptives on the activity of the hypothalamus-pituitaryadrenal axis. Psychosomatic Medicine 199961 154-162. (https://doi. org/10.1097/00006842-199903000-00006)

23 Skeldon AC, Derks G \& Dijk D-J. Modelling changes in sleep timing and duration across the lifespan: changes in circadian rhythmicity or sleep homeostasis? Sleep Medicine Reviews 201628 96-107. (https:// doi.org/10.1016/j.smrv.2015.05.011)

24 Palagini L, Baglioni C, Ciapparelli A, Gemignani A \& Riemann D. REM sleep dysregulation in depression: state of the art. Sleep Medicine Reviews 201317 377-390. (https://doi.org/10.1016/j. smrv.2012.11.001)

25 Sheehan DV, Lecrubier Y, Sheehan KH, Amorim P, Janavs J, Weiller E, Hergueta T, Baker R \& Dunbar GC. The Mini-International Neuropsychiatric Interview (M.I.N.I.): the development and validation of a structured diagnostic psychiatric interview for DSM-IV and ICD-10. Journal of Clinical Psychiatry 199859 (Supplement 20) 22-33.

26 Beck AT, Steer RA \& Brown GK. Beck Depression Inventory Manual. San Antonio, TX: Psychological Corporation, 1996.

27 Buysse DJ, Reynolds CF III, Monk TH, Berman SR \& Kupfer DJ. The Pittsburgh Sleep Quality Index: a new instrument for psychiatric practice and research. Psychiatric Research 198928 193-213. (https:// doi.org/10.1016/0165-1781(89)90047-4)

28 Mollayeva T, Thurairajah P, Burton K, Mollayeva S, Shapiro CM \& Colantonio A. The Pittsburgh Sleep Quality Index as a screening tool for sleep dysfunction in clinical and non-clinical samples: a systematic review and meta-analysis. Sleep Medicine Reviews 201625 52-73. (https://doi.org/10.1016/j.smrv.2015.01.009)

29 Kushida CA, Chang A, Gadkary C, Guilleminault C, Carrillo O \& Dement WC. Comparison of actigraphic, polysomnographic, and subjective assessment of sleep parameters in sleep-disordered patients. Sleep Medicine 20012 389-396. (https://doi.org/10.1016/ S1389-9457(00)00098-8)

30 Berry RB, Brooks R, Gamaldo CE, Harding SM, Marcus C \& Vaughn B. The AASM manual for the scoring of sleep and associated events. In. Rules, Terminology and Technical Specifications. Darien, Illinois: American Academy of Sleep Medicine, 2012.

31 World Medical Association. World Medical Association Declaration of Helsinki: ethical principles for medical research involving human subjects. JAMA 2013310 2191-2194. (https://doi.org/10.1001/ jama.2013.281053)

32 Markovic A, Achermann P, Rusterholz T \& Tarokh L. Heritability of sleep EEG topography in adolescence: results from a longitudinal Twin Study. Scientific Reports 20188 7334. (https://doi.org/10.1038/ s41598-018-25590-7)

33 Roehrs T \& Roth T. Insomnia as a path to alcoholism: tolerance development and dose escalation. Sleep 201841 zsy091. (https://doi. org/10.1093/sleep/zsy091)

34 Sprajcer M, Jay SM, Vincent GE, Vakulin A, Lack L \& Ferguson SA. The effects of anticipating a high-stress task on sleep and performance during simulated on-call work. Journal of Sleep Research 2018 e12691. (https://doi.org/10.1111/jsr.12691)

35 Erdfelder E, Faul F \& Gpower BA. GPOWER: a general power analysis program. Behavior Research Methods, Instruments and Computers 1996 28 1-11. (https://doi.org/10.3758/BF03203630)

36 Diekelmann S \& Born J. The memory function of sleep. Nature Reviews: Neuroscience 201011 114-126. (https://doi.org/10.1038/ nrn2762)

37 Luyster FS, Strollo PJ, Zee PC, Walsh JK \& Boards of Directors of the American Academy of Sleep Medicine and the Sleep Research Society. Sleep: a health imperative. Sleep 201235 727-734. (https://doi. org/10.5665/sleep.1846) 
38 Depner CM, Stothard ER \& Wright KP Jr. Metabolic consequences of sleep and circadian disorders. Current Diabetes Reports 201414507. (https://doi.org/10.1007/s11892-014-0507-z)

39 Zhi T-F, Sun X-M, Li S-J, Wang Q-S, Cai J, Li L-Z, Li Y-X, Xu M-J, Wang Y, Chu X-F et al. Associations of sleep duration and sleep quality with life satisfaction in elderly Chinese: the mediating role of depression Chinese. Archives of Gerontology and Geriatrics $2016 \mathbf{6 5}$ 211-217. (https://doi.org/10.1016/j.archger.2016.03.023)

40 Lovas K \& Husebye ES. Replacement therapy in Addison's disease. Expert Opinion on Pharmacotherapy 20034 2145-2149. (https://doi. org/10.1517/14656566.4.12.2145)

41 Johannsson G, Falorni A, Skrtic S, Lennernäs H, Quinkler M, Monson JP \& Stewart PM. Adrenal insufficiency: review of clinical outcomes with current glucocorticoid replacement therapy. Clinical Endocrinology 201582 2-11. (https://doi.org/10.1111/ cen.12603)
42 Bennion KA, Mickley Steinmetz KR, Kensinger EA \& Payne JD. Sleep and cortisol interact to support memory consolidation. Cerebral Cortex 201525 646-657. (https://doi.org/10.1093/cercor/bht255)

43 Antonijevic IA \& Steiger A. Depression-like changes of the sleep-EEG during high dose corticosteroid treatment in patients with multiple sclerosis. Psychoneuroendocrinology 200328 780-795. (https://doi. org/10.1016/S0306-4530(02)00085-9)

44 Del Corral P, Schurman R, Kinza S, Fitzgerald M, Kordick C, Rusch J \& Nadolski J. Salivary but not plasma cortisone tracks the plasma cortisol response to exercise: effect of time of day. Journal of Endocrinological Investigation 201639 315-322. (https://doi. org/10.1007/s40618-015-0367-7)

45 Lipinska G \& Thomas KG. Better sleep in a strange bed? Sleep quality in South African women with posttraumatic stress disorder. Frontiers in Psychology 20178 1555. (https://doi.org/10.3389/ fpsyg.2017.01555)

Received 23 May 2018

Revised version received 17 July 2018

Accepted 14 August 2018 